\title{
Major bleeding complications in critically ill patients with COVID-19 pneumonia
}

\author{
Anne Godier ${ }^{1,2,4}\left(D^{1} \cdot\right.$ Darless Clausse $^{1} \cdot$ Simon Meslin $^{1} \cdot$ Myriame Bazine $^{1} \cdot$ Elodie Lang $^{1} \cdot$ Florian Huche $^{1} \cdot$ \\ Bernard Cholley ${ }^{1,2} \cdot$ Sophie Rym Hamada ${ }^{1,3}$
}

Accepted: 15 February 2021 / Published online: 1 March 2021

(C) The Author(s), under exclusive licence to Springer Science+Business Media, LLC part of Springer Nature 2021

\begin{abstract}
As patients with COVID-19 pneumonia admitted to intensive care unit (ICU) have high rates of thrombosis, high doses of thromboprophylaxis have been proposed. The associated bleeding risk remains unknown. We investigated major bleeding complications in ICU COVID-19 patients and we examined their relationship with inflammation and thromboprophylaxis. Retrospective monocentric study of consecutive adult patients admitted in ICU for COVID-19 pneumonia requiring mechanical ventilation. Data collected included demographics, anticoagulation status, coagulation tests and outcomes including major bleeding and thrombotic events. Among 56 ICU COVID-19 patients, 10 (18\%) patients had major bleeding and $16(29 \%)$ thrombotic events. Major bleeding occurred later than thrombosis after ICU admission [17(14-23) days versus 9(3-11) days respectively $(\mathrm{p}=0.005)]$. Fibrinogen concentration always decreased several days [4(3-5) days] before bleeding; D-dimers followed the same trend. All bleeding patients were treated with anticoagulants and anticoagulation was overdosed for 6 $(60 \%)$ patients on the day of bleeding or the day before. In the whole cohort, overdose was measured in 22 and $78 \%$ of patients receiving therapeutic anticoagulation during fibrinogen increase and decrease respectively $(\mathrm{p}<0.05)$. Coagulation disorders had biphasic evolution during COVID-19: first thrombotic events during initial hyperinflammation, then bleeding events once inflammation reduced, as confirmed by fibrinogen and D-dimers decrease. Most bleeding events complicated heparin overdose, promoted by inflammation decrease, suggesting to carefully monitor heparin during COVID-19. Thromboprophylaxis may be adapted to this biphasic evolution, with initial high doses reduced to standard doses once the high thrombotic risk period ends and fibrinogen decreases, to prevent bleeding events.
\end{abstract}

Keywords Anticoagulant $\cdot$ Bleeding $\cdot$ COVID- $19 \cdot$ Fibrinogen $\cdot$ Inflammation $\cdot$ Thrombosis

\section{Highlights}

- Increased thromboprophylaxis is proposed to COVID-19 patients to prevent thrombosis

Anne Godier

anne.godier@aphp.fr

1 Department of Anaesthesiology and Critical Care, European Georges Pompidou Hospital, Assistance Publique-Hôpitaux de Paris, AP-HP, Université de Paris, Paris, France

2 INSERM UMRS-1140, Université de Paris, Paris, France

3 CESP, Inserm, Maison de Solenn, Université Paris Sud, Université Paris-Saclay, Paris, France

4 Service D’Anesthésie-Réanimation, Hôpital Pompidou, 20 rue Leblanc, 75015 Paris, France
- We assessed the bleeding risk of critically ill patients with COVID-19 pneumonia

- Bleeding occurred later than thrombosis

- Bleeding occurred once inflammation decreased thus favored heparin overdose

- We may reduce thromboprophylaxis doses once inflammation corrects to prevent bleeding

\section{Introduction}

Patients with severe pneumonia due to SARS-CoV-2 infection, also known as coronavirus disease 2019 (COVID-19), who are admitted to intensive care units (ICUs) have high rates of thrombosis, particularly pulmonary embolism [1]. There is a growing body of evidence that severe COVID19 is associated with a prothrombotic state resulting from 
endothelial dysfunction and hyperinflammation, and characterized by high levels of D-dimers and fibrinogen [2,3]. Therefore, pharmacologic thromboprophylaxis is recommended. Some experts advocate intensification of anticoagulation, from prophylactic to intermediate or even therapeutic doses for high risk patients $[4,5]$. However, the associated bleeding risk is unknown. The goal of this study was to investigate major bleeding complications in a cohort of critically ill patients with COVID-19 pneumonia and to examine their relationship with inflammation and thromboprophylaxis.

\section{Methods}

We conducted a retrospective chart review of all adult patients admitted to our ICU for laboratory-confirmed COVID-19 pneumonia requiring mechanical ventilation between 20 March and 14 April 2020. This study was approved by the Institutional Review Board of AssistancePublique Hôpitaux-de-Paris Centre (Ref.2020-CVD-15) and informed consent was obtained from all participants or next of kin.

Pharmacological thromboprophylaxis was prescribed according to the GIHP guidance document [5]: patients received high prophylactic anticoagulation (enoxaparin $4000 \mathrm{IU} / 12 \mathrm{~h} \mathrm{SC}$ or $6000 \mathrm{IU} / 12 \mathrm{~h} \mathrm{SC}$ if weight $>120 \mathrm{~kg}$, or UFH in case of renal insufficiency). The anticoagulation level was increased to curative dose in the following situations: patients with a BMI $>30 \mathrm{~kg} / \mathrm{m}^{2}$ and known risk factors for venous thromboembolism, long-term anticoagulant therapy, catheter or iterative filter coagulation, severe inflammatory syndrome (e.g. fibrinogen $>8 \mathrm{~g} / \mathrm{l}$ ), hypercoagulable state (e.g. D-dimer $>3.0 \mu \mathrm{g} / \mathrm{ml}$ ) and ECMO.

Data collected included demographics, relevant comorbidities, anticoagulation status, results of coagulation tests and outcomes including major bleeding events, thrombotic events, length of stay and mortality. Outcomes were assessed from ICU admission to ICU discharge or death.

Major bleeding was defined according to the International Society on Thrombosis and Haemostasis as overt bleeding among the following: fatal bleeding, symptomatic bleeding in a critical area or organ (i.e., intracranial, intraspinal, intraocular, retroperitoneal, intraarticular, pericardial, intramuscular with compartment syndrome), bleeding causing a fall in hemoglobin level of $20 \mathrm{~g} / \mathrm{l}$ or more or leading to transfusion of two or more units of whole blood or red cells [6].

Thrombotic events included deep vein thrombosis or pulmonary embolism confirmed with imaging, limb ischemia, ischemic stroke and clinically significant non-vessel thrombotic complications (clotting of extracorporeal membrane oxygenation, iterative clotting of central venous or arterial catheter or circuits of renal replacement therapies).
Supratherapeutic anticoagulation (overdose) was defined as anti-Xa activity $>0.7 \mathrm{UI} / \mathrm{ml}$ for unfractionated heparin (UFH) and $>1 \mathrm{UI} / \mathrm{ml}$ for low molecular weight heparin (LMWH) respectively.

\section{Statistical analysis}

Descriptive results are expressed as median [quartile 1, 3] or count (percentage) and were compared using Wilcoxon and Chi2/Fisher test. $\mathrm{p}<0.05$ was considered significant. (BiostaTGV, France).

\section{Results}

Fifty-six patients with confirmed COVID-19 pneumonia were included. They were mostly men $(n=40,71 \%)$, with a median age of 60 [53-69] years and increased body mass index [27 (24-31) kg/m ${ }^{2}$. All patients underwent invasive mechanical ventilation, with prone positioning in 39 patients (70\%). The lowest $\mathrm{PaO}_{2} / \mathrm{FiO}_{2}$ ratio within the ICU stay was 76 [59-107] mmHg. Seven patients (13\%) were treated with extracorporeal membrane oxygenation (ECMO). The simplified acute physiology score (SAPS) II was 43 [40;49]. All patients were discharged at the time of data analysis.

Sixteen (29\%) patients had thrombotic events whereas 10 (18\%) patients had major bleeding, including the 7 patients with ECMO. Major bleeding events included 3 deep muscle hematomas, 3 gastrointestinal bleeding events, 3 vascular catheter bleeding events and one intracranial hemorrhage. Thrombotic events and bleeding events occurred 9 [3-11] days and 17 [14-23] days after ICU admission respectively $(\mathrm{p}=0.005)$ (Fig. 1).

Fibrinogen concentrations and D-dimers had the same biphasic evolution: they were high in all patients at ICU admission: 7 [6-7.6] g/l and 1401 [1056-2122] $\mathrm{ng} / \mathrm{ml}$ respectively; they still increased to reach 8.5 [7.4-9.3] g/l and 5908 [3544-9380] $\mathrm{ng} / \mathrm{ml}$ on day 5 [2-9] and day 6 [3-8] $(p=0.33)$ respectively; then, they progressively decreased.

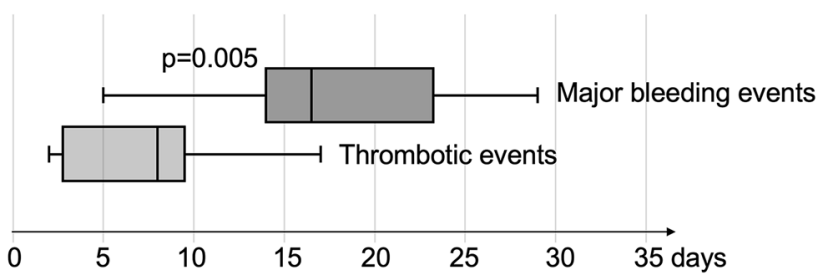

Fig. 1 Time from ICU admission to major bleeding events and thrombotic events in critically ill COVID-19 patients. Box-andwhisker plots showing median, 25 and $75 \%$ percentile, minimum and maximum. Comparison between time to major bleeding events and time to thrombotic events showed that major bleeding events occurred significantly later $(\mathrm{p}=0.005)$ 
During fibrinogen increase, $22 \%$ of the 36 patients receiving therapeutic anticoagulation had anticoagulant overdose, compared to $78 \%(n=28)$ of them once fibrinogen decreased $(\mathrm{p}<0.05)$. Anticoagulant overdose was measured 7.5 [3.5-9.25] days after fibrinogen peak.

The 10 major bleeding events occurred while inflammation was decreasing, after 4 [3-5] days of fibrinogen decrease (Fig. 2). Similarly, D-dimers were decreasing in $86 \%$ of the bleeding patients that had 2 measures of D-dimers or more within the week prior the bleeding event. All patients with major bleeding were treated with anticoagulants $(\mathrm{UFH} \mathrm{n}=7$; LMWH $\mathrm{n}=3$ ), all but one at therapeutic doses. Anticoagulation was supratherapeutic for $6(60 \%)$ patients on the day of bleeding or the day before (Fig. 2). Half of the bleeding patients died in ICU, compared to $37 \%$ of patients without bleeding complications (NS).

\section{Discussion}

We reported that major bleeding was common in this series of critically ill patients with COVID-19 pneumonia. Most of the major bleeding events complicated therapeutic anticoagulation, as previously observed in a cohort of 92 ICU patients where the rate of bleeding events in ICU was $21 \%$, with $84 \%$ during therapeutic anticoagulation [7]. Moreover, more than half of our bleeding complications occurred while heparin, initially in therapeutic ranges, became supratherapeutic. This might be explained by the decrease in inflammation: indeed, heparin binds to antithrombin to mediate anticoagulant effects but also binds nonspecifically to other plasma proteins that compete with antithrombin. During the hyperinflammation state, markedly increased levels of acute-phase-reactant proteins, including fibrinogen, contribute to heparin resistance [8]. Then, decreased concentrations of inflammatory proteins, including fibrinogen, induce increased anticoagulant effect and potential overdose. These data highlight to carefully monitor heparin in COVID19 patients, using anti-Xa activity preferentially, to prevent under and overdosage, especially once the initial hyperinflammation decreases.

As we recorded data until ICU discharge, we observed a biphasic evolution of coagulation disorders during COVID19: first thrombotic events during the early hyperinflammation phase, then bleeding events once inflammation reduced, as confirmed by fibrinogen and D-dimers decrease. Although more studies are needed to determine when the thrombotic risk period ends, these data suggest that intensity of thromboprophylaxis may be adapted to the kinetics of thromboinflammation: intensification of anticoagulation doses may be used during the initial hyperinflammation phase as proposed by many experts $[4,5,9]$, then standard prophylaxis may be considered once the high thrombotic risk period ends and fibrinogen decreases, to prevent bleeding events.

\section{Conclusion}

Despite the limitations associated with the retrospective monocenter design of our study, these data highlighted the relationship between fibrinogen level, inflammation and risk of thrombosis or bleeding and the evolution of the risks during COVID-19 hospitalization. Bleeding is likely to occur later during ICU stay when inflammation is decreasing and exposes to supra-therapeutic levels of anticoagulation. Intensivists may modulate thromboprophylaxis strategy according to inflammatory biomarkers, especially fibrinogen.
Fig. 2 Changes of fibrinogen concentration in the 10 critically ill COVID-19 patients facing major bleeding events. Each line represents the fibrinogen concentrations of one patient. Fibrinogen concentrations are shown from ICU admission (first point of each line on the left) to major bleeding events (black arrow). All bleeding events occurred after several days of fibrinogen decrease [4 (3-5) days]. Red circles (O) represent supratherapeutic anticoagulation observed the day of bleeding or the day before ( 6 patients over 10)

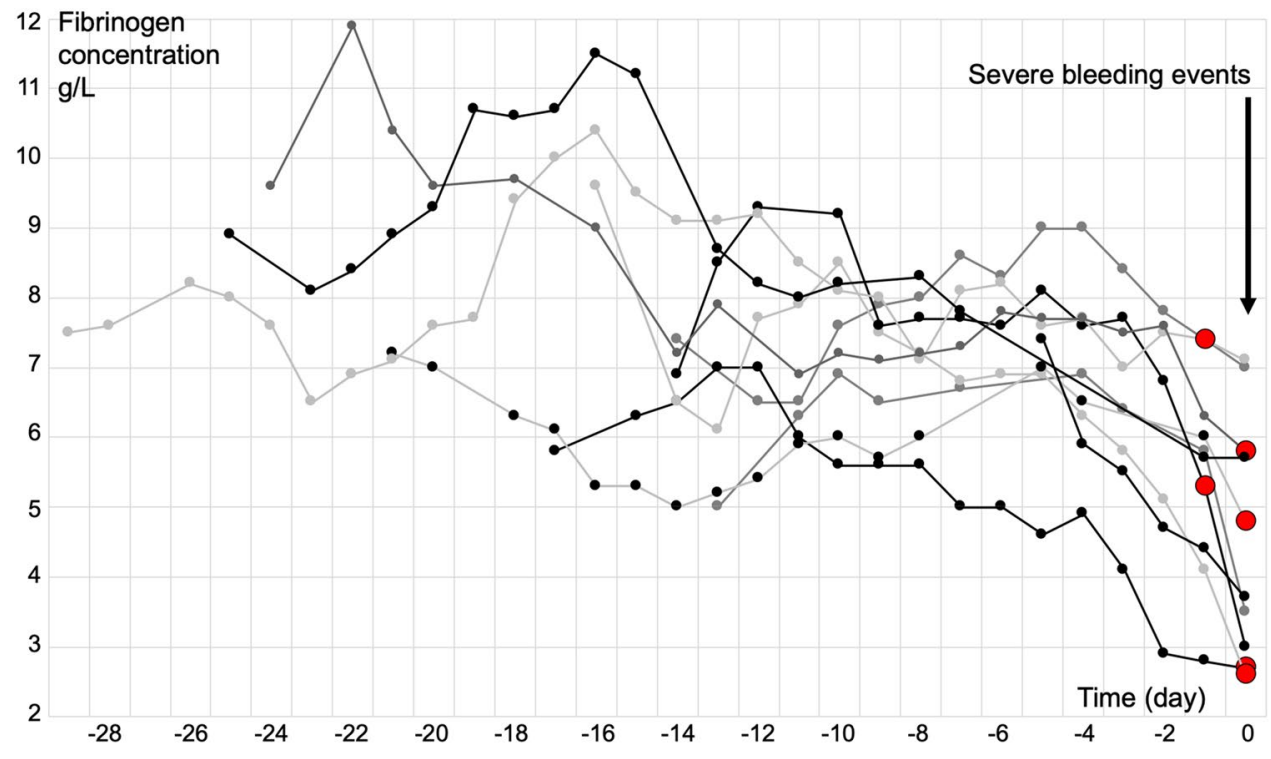


Moreover, when fibrinogen decreases repeatedly, anti-Xa activity should be controlled to adapt heparin dosing.

Author contributions $\mathrm{AG}, \mathrm{BC}$ and $\mathrm{SH}$ designed research, DC, SM, $\mathrm{MB}$, EL, FH performed research, DC, SM, BC analyzed data, and AG, $\mathrm{SH}, \mathrm{SM}$ wrote the first draft, MB, EL, FH, BC corrected the paper.

Funding This research did not receive any specific grant from funding agencies in the public, commercial, or not-for-profit sectors.

Data availability Available on request of the editor.

\section{Declarations}

Conflict of interest AGo: honoraria and travel fees from Bayer-Healthcare, Boehringer-Ingelheim, Bristol-Myers-Squibb/Pfizer and Sanofi. Other authors declares that they have no conflict of interest.

Ethical approval This study was approved by the Institutional Review Board of Assistance-Publique Hôpitaux-de-Paris Centre (Ref.2020CVD-15) and informed consent was obtained from all participants or next of kin.

Informed consent This study was approved by the Institutional Review Board of Assistance-Publique Hôpitaux-de-Paris Centre (Ref.2020CVD-15) and informed consent was obtained from all participants or next of kin. Informed consent is for both participation to this observational study and publication.

\section{References}

1. Poissy J, Goutay J, Caplan M, Parmentier E, Duburcq T, Lassalle F, Jeanpierre E, Rauch A, Labreuche J, Susen S, Lille ICU Haemostasis COVID-19 Group (2020) Pulmonary embolism in patients with COVID-19: awareness of an increased prevalence. Circulation 142:184-186

2. Ackermann M, Verleden SE, Kuehnel M, Haverich A, Welte T, Laenger F, Vanstapel A, Werlein C, Stark H, Tzankov A, Li
WW, Li VW, Mentzer SJ, Jonigk D (2020) Pulmonary vascular endothelialitis, thrombosis, and angiogenesis in COVID-19. N Engl J Med 383:120-128

3. Rauch A, Labreuche J, Lassalle F, Goutay J, Caplan M, Charbonnier L, Rohn A, Jeanpierre E, Dupont A, Duhamel A, Faure K, Lambert M, Kipnis E, Garrigue D, Lenting PJ, Poissy J, Susen $S$ (2020) Coagulation biomarkers are independent predictors of increased oxygen requirements in COVID-19. J Thromb Haemost 18(11):2942-2953. https://doi.org/10.1111/jth.15067

4. Rosovsky RP, Sanfilippo KM, Wang TF, Rajan SK, Shah S, Martin KA, Ní Áinle F, Huisman M, Hunt BJ, Kahn SR, Kevane B, Lee AY, McLintock C, Baumann KL (2020) Anticoagulation practice patterns in COVID-19: a global survey. Res Pract Thromb Haemost 4:969-983

5. Susen S, Tacquard CA, Godon A, Mansour A, Garrigue D, Nguyen P, Godier A, Testa S, Levy JH, Albaladejo P, Gruel Y (2020) Prevention of thrombotic risk in hospitalized patients with COVID-19 and hemostasis monitoring. Crit Care 24:364

6. Schulman S, Kearon C, Subcommittee on Control of Anticoagulation of the Scientific and Standardization Committee of the International Society on Thrombosis and Haemostasis (2005) Definition of major bleeding in clinical investigations of antihemostatic medicinal products in non-surgical patients. J Thromb Haemost 3:692-694

7. Fraissé M, Logre E, Pajot O, Mentec H, Plantefève G, Contou D (2020) Thrombotic and hemorrhagic events in critically ill COVID-19 patients: a French monocenter retrospective study. Crit Care 24:275

8. Young E, Podor TJ, Venner T, Hirsh J (1997) Induction of the acute-phase reaction increases heparin-binding proteins in plasma. Arterioscler Thromb Vasc Biol 17:1568-1574

9. Al-Samkari H, Karp Leaf RS, Dzik WH, Carlson JCT, Fogerty AE, Waheed A, Goodarzi K, Bendapudi PK, Bornikova L, Gupta S, Leaf DE, Kuter DJ, Rosovsky RP (2020) COVID-19 and coagulation: bleeding and thrombotic manifestations of SARS-CoV-2 infection. Blood 136:489-500

Publisher's Note Springer Nature remains neutral with regard to jurisdictional claims in published maps and institutional affiliations. 